\title{
Depletion of Bmi-1 enhances 5-fluorouracil-induced apoptosis and autophagy in hepatocellular carcinoma cells
}

\author{
JING WU, DONG HU and RONGBO ZHANG
}

Department of Medical Immunology, School of Medicine, Anhui University of Science and Technology, Huainan, Anhui 232001, P.R. China

Received March 6, 2012; Accepted June 14, 2012

DOI: $10.3892 / \mathrm{ol} .2012 .805$

\begin{abstract}
FU) is one of the standard chemoradiotherapy regimens for hepatocellular carcinoma (HCC) treatment. B-cell-specific Moloney murine leukemia virus insertion site 1 (Bmi-1) has been demonstrated to regulate proliferation. Additionally, Bmi-1 overexpression has been identified in HCC cell lines and correlates with the advanced invasive stage of tumor progression and poor prognosis. In this study, we examined the effects of 5-FU treatment on cell growth in HCC cells with or without Bmi-1 depletion. The $\mathrm{IC}_{50}$ values of 5-FU were significantly decreased to a greater extent in cells with Bmi-1 knockdown. Depletion of Bmi-1 increased sensitivity of the cells to 5-FU and increased apoptosis. Knockdown of endogenous Bmi-1 led to a substantial reduction in the levels of phospho-AKT and Bcl-2 with a concomitant increase in the levels of Bax. Additionally, 5-FU induced the conversion/turnover of microtubule-associated protein 1 light chain 3 (LC3). Knockdown of endogenous Bmi-1 led to an increase in the levels of Beclin-1 and the accumulation of LC3-II. Together, these findings reveal that Bmi-1 depletion enhanced the chemosensitivity of HCC cells by inducing apoptosis and autophagy, which is associated with the PI3K/AKT and Bcl-2/Beclin-1 pathways.
\end{abstract}

\section{Introduction}

Hepatocellular carcinoma (HCC) is the third most common cause of cancer-related mortality worldwide, accounting for approximately 500,000 mortalities annually. The majority of liver-related cancer cases occur in Asia and Africa (1). HCC has a long latency period, but is often diagnosed at late stages

Correspondence to: Dr Dong Hu or Professor Rongbo Zhang, Department of Medical Immunology, School of Medicine, Anhui University of Science and Technology, No. 25 Middle Dongshan Road, Huainan, Anhui 232001, P.R. China

E-mail: austhudong@126.com

E-mail: lory456@126.com

Key words: B-cell-specific Moloney murine leukemia virus insertion site 1, 5-fluorouracil, hepatocellular carcinoma, apoptosis, autophagy when the tumors are of high grade and progress rapidly. At present, only one third of newly diagnosed HCC patients are eligible for potential curative therapies, including hepatic resection, liver transplantation, transcatheter arterial chemoembolization or radiofrequency ablation (2).

Despite the uncertain clinical benefits, patients with advanced disease are routinely treated with transcatheter arterial chemoembolization and systemic chemotherapy using 5-fluorouracil (5-FU), doxorubicin, cisplatin or interferon (3). However, a sizable proportion of HCC patients do not respond to the cytotoxic effects of 5-FU treatment, which is mainly due to drug-resistant cancer cells. Therefore, new strategies for enhancing the sensitivity of cancer cells to drug-induced apoptosis for cancer therapy have been intensively explored.

Polycomb group $(\mathrm{PcG})$ proteins are epigenetic chromatin modifiers involved in cancer development. The B-cell-specific Moloney murine leukemia virus insertion site 1 gene (Bmi-1), the first PcG gene to be identified, was originally discovered as an oncogene that cooperated with c-Myc in the initiation of lymphoma in murine models (4-6). Bmi-1 is overexpressed in several carcinomas, including liver carcinoma (7). Overexpression of Bmi-1 was also observed in a significant number of HCC cases, and was correlated with advanced invasive stage of tumor progression and poor prognosis (8). Recently, it has been reported that the downregulation of Bmi-1 may result in apoptosis of cancer cells (9). Therefore, we hypothesize that the abrogation of Bmi-1 expression may be an effective strategy for sensitizing human cancer cells, including HCC cells, to cancer chemotherapy.

The present study was designed to demonstrate the in vitro anticancer effects of the combination of 5-FU treatment and Bmi-1 depletion on hepatoma cells (SK-HEP-1 and SMMC-7721), with an emphasis on their in vitro molecular targets. Our results suggest that the combination of 5-FU treatment and Bmi-1 depletion may be a potential clinical strategy for cancer chemotherapy.

\section{Materials and methods}

Cell culture. The HCC cell lines SK-HEP-1 and SMMC-7721 were maintained in Dulbecco's modified Eagle's medium (DMEM; Gibco, Invitrogen Corporation, Carlsbad, CA, USA), supplemented with $10 \%$ fetal bovine serum (Gibco), $100 \mathrm{U} / \mathrm{ml}$ penicillin and $100 \mathrm{U} / \mathrm{ml}$ streptomycin 
(Gibco), and cultured at $37^{\circ} \mathrm{C}$ in a $5 \% \mathrm{CO}_{2}$ humidified incubator.

Gene knockdown. To knockdown Bmi-1, SK-HEP-1 and SMMC-7721 cell lines were transfected with $50 \mathrm{nmol} / 1$ of Bmi-1 targeted small interfering RNA (siRNA) oligonucleotides (siBmi1; 5'-AAAUGGACAUACCUAAUAC-3') or negative control siRNA oligonucleotides (siNS; 5'-ACGCATGCATGCTTGCTTT-3') (Invitrogen Life Technologies).

Measurement of cytotoxicity. SK-HEP-1 $\left(2 \times 10^{3}\right)$ and SMMC-7721 $\left(3 \times 10^{3}\right)$ cells were seeded in $200 \mu 1$ DMEM into 96-well plates and cultured overnight. SK-HEP-1 and SMMC-7721 cells were then treated with various concentrations of 5-FU (Sigma-Aldrich, St. Louis, MO, USA) for $72 \mathrm{~h}$. At the end of the treatment, 3-(4,5-dimethylthiazol-2-yl)-2,5-diphenyltetrazolium bromide (MTT) was added into each well at a final concentration of $0.5 \mathrm{mg} / \mathrm{ml}$, which was followed by incubation at $37^{\circ} \mathrm{C}$ for $3 \mathrm{~h}$ in the dark. The culture medium containing MTT was discarded and the dye crystals were dissolved in dimethyl sulfoxide (DMSO). The viable cells were detected by reading the absorbance of the metabolic MTT at wavelength $570 \mathrm{~nm}$. All experiments were conducted in triplicate.

Quantification ofapoptoticcells.Following the manufacturer's instructions, flow cytometry using the Annexin V-Fluorescein Isothiocyanate (FITC) Apoptosis Detection kit was used to determine 5-FU treatment-induced apoptosis in HCC cells treated with 5-FU alone and in combination with Bmi-1 depletion. Briefly, $2 \times 10^{5}$ cells were treated with 5-FU alone or in combination with Bmi-1 depletion for $72 \mathrm{~h}$. The cells were harvested, washed in phosphate-buffered saline and then incubated with Annexin V and propidum iodide for staining in binding buffer at room temperature for $10 \mathrm{~min}$ in the dark. The stained cells were analyzed using a Becton Dickinson FACSCalibur flow cytometer (BD Biosciences, San Jose, CA, USA).

Western blot analysis. Western blot analysis was performed (10) and the following antibodies were used: anti-Bmi-1 (R\&D Systems, Minneapolis, MN, USA), anti-light chain 3 B (LC3) (Novus Biologicals, Littleton, CO, USA), anti-phospho-AKT, anti-Bcl2, anti-BAX (Santa Cruz Biotechnology, Inc., Santa Cruz, CA, USA), anti-Beclin-1, anti- $\beta$-actin and secondary antibodies (Proteintech Group, Inc., Chicago, IL, USA).

\section{Results}

Bmi-1 knockdown sensitizes cells to 5-FU. siBmil was examined for silencing in SK-HEP-1 and SMMC-7721 cells. Western blot analysis was used to confirm that siBmil was able to reduce Bmi-1 protein expression in SK-HEP-1 cells and SMMC-7721 cells (Fig. 1A and B).

Once the SK-HEP-1-siBmi1 (SK-siBmi1), SK-HEP-1-siNS (SK-siNS), SMMC-7721-siBmi1 (7721-siBmi1) and SMMC-7721-siNS (7721-siNS) cells were treated with 5-FU (1-320 mg/l) for $72 \mathrm{~h}$, an MTT assay was performed to examine
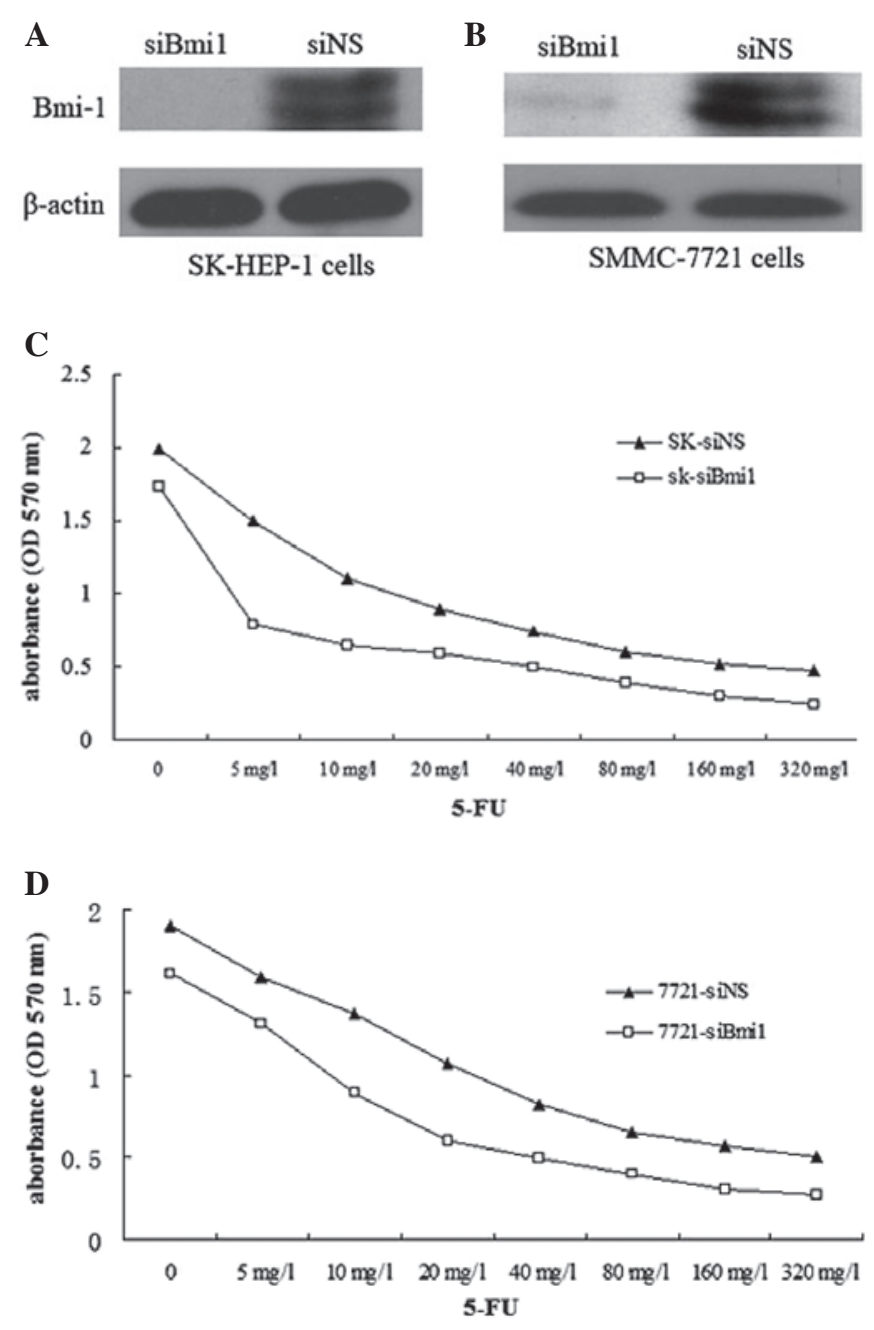

Figure 1. Effect of Bmi-1 downregulation on the chemosensitivity of SK-HEP-1 and SMMC-7721 cells to 5-FU treatment. (A and B) Effect of siBmil in SK-HEP-1 and SMMC-7721 cells. Protein samples were isolated from SK-HEP-1-siBmi1 (SK-siBmi1), SK-HEP-1-siNS (SK-siNS), SMMC-7721-siBmil (7721-siBmi1) and SMMC-7721-siNS (7721-siNS) cells The protein expression of Bmi-1 was detected using western blot analysis. Triplicate experiments revealed consistent results. (C and D) Cell viability was determined using an MTT assay. SK-siBmi1, SK-siNS, 7721-siBmi1 and 7721-siNS cells were treated with increasing concentrations of 5-FU for $72 \mathrm{~h}$. All assays were conducted in triplicate. Bmi-1, B-cell-specific Moloney murine leukemia virus insertion site 1; siBmil, Bmi-1 targeted siRNA oligonucleotide; siNS, negative control siRNA oligonucleotides; OD, optical density; 5-FU, 5-fluorouracil.

the effect of 5-FU on the survival of Bmi-1 knockdown cells. The $\mathrm{IC}_{50}$ values of 5-FU in the SK-siBmil and SK-siNS cells were 9.97 and $20.85 \mathrm{mg} / \mathrm{l}$, respectively; while the $\mathrm{IC}_{50}$ values of 5-FU in 7721-siBmil and 7721-siNS cells were 15.39 and $27.11 \mathrm{mg} / 1$, respectively (Fig. 1C and D). The results indicated that Bmi-1 depletion increased the sensitivity of the cells to 5 -FU.

Depletion of Bmi-1 enhances 5-FU-induced apoptosis. The combination of 5-FU with Bmi-1 knockdown resulted in a significant reduction in cell viability compared to the treatment with 5-FU alone in the cells. As demonstrated in Fig 1, depletion of Bmi-1 increased the sensitivity of the cells to 5-FU in SK-HEP-1 cells compared to SMMC-7721; thus, we selected 


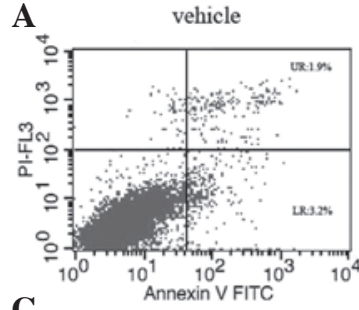

C
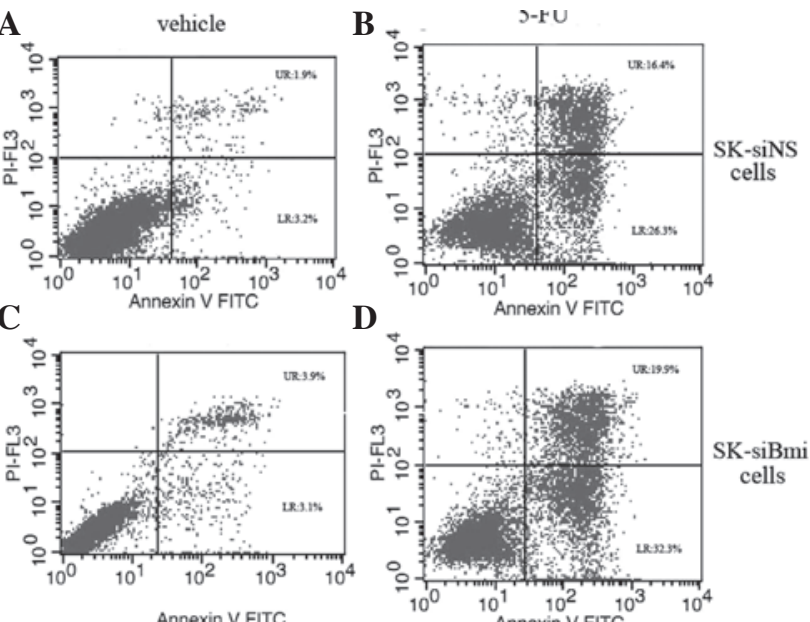

D

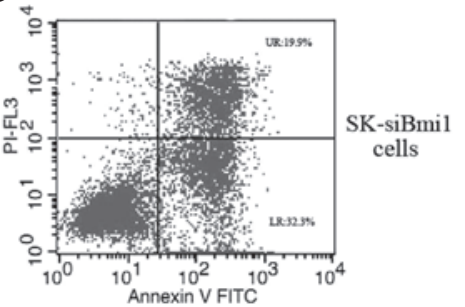

Figure 2. Effect of Bmi-1 knockdown on 5-FU-induced apoptosis. Flow cytometry was performed to measure apoptotic rates. Percentages of apoptotic cells were identified in (A) untreated SK-siNS, (B) SK-siNS cells treated with $10 \mathrm{mg} / 1$ 5-FU for $72 \mathrm{~h}$, (C) untreated SK-siBmil and (D) SK-siBmi1 cells treated with $10 \mathrm{mg} / 1$ 5-FU for $72 \mathrm{~h}$. 5-FU, 5 -fluorouracil; FITC, fluorescein isothiocyanate; siNS, negative control siRNA oligonucleotides; siBmi1, Bmi-1 targeted siRNA oligonucleotide; Bmi-1, B-cell-specific Moloney murine leukemia virus insertion site 1 .

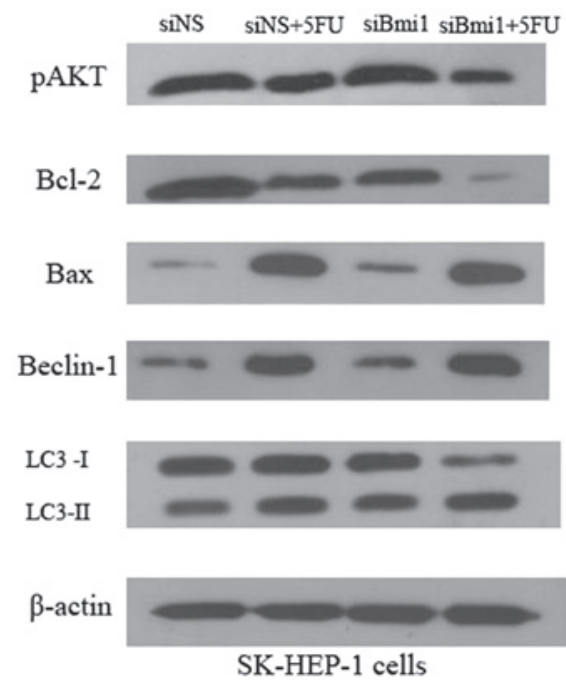

Figure 3. Effects of depletion of Bmi-1 expression on p-AKT, Bcl-2, Bax, Beclin-1 and LC3 levels. SK-siNS and SK-siBmil cells were incubated for $72 \mathrm{~h}$ with or without 5-FU treatment. The protein expression of p-AKT, Bcl-2, Bax, Beclin-1 and LC3 was detected by western blot analysis. All assays were conducted in triplicate. siNS, negative control siRNA oligonucleotides; 5-FU, 5-fluorouracil; siBmi1, Bmi-1 targeted siRNA oligonucleotide; Bmi-1, B-cell-specific Moloney murine leukemia virus insertion site 1 ; LC3, light chain 3 .

SK-HEP-1 cells for further investigations. In order to evaluate the effect of Bmi-1 depletion on the induction of apoptosis, the SK-siBmil and SK-siNS cells were treated with $10 \mathrm{mg} / \mathrm{l}$ of 5 -FU for $72 \mathrm{~h}$. Flow cytometry data revealed that the apoptotic rate among the SK-siBmil cells was $51 \%$ compared to $42 \%$ among the SK-siNS cells (Fig. 2).

Knockdown of Bmi-1 inhibits AKT activation and regulates expression of apoptosis-related proteins Bcl-2 and Bax in
SK-HEP-1 cells. Bcl-2 family proteins play critical roles in the regulation of apoptosis (11). To further explore the mechanism underlying the enhancement of 5-FU-induced apoptosis by the silencing of Bmi-1, we examined the expression levels of phospho-AKT, Bax and Bcl-2 in SK-siBmi-1 and SK-siNS cells. Western blot analysis demonstrated that knockdown of endogenous Bmi-1 led to a substantial reduction in the levels of phospho-AKT. Consistent with this reduction in the phospho-AKT level, the results identified a significant decreased expression of Bcl-2 in Bmi-1 knockdown cells exposed to 5-FU and an increase in the level of Bax (Fig. 3).

5-FU triggers autophagy and depletion of Bmi-1 enhanced 5-FU-induced autophagy in SK-HEP-1 cells. To investigate whether 5-FU triggers autophagy in SK-HEP-1 cells, and whether depletion of Bmi-1 enhances 5-FU-induced apoptosis with a concomitantly induced autophagy, we examined the expression of Beclin-1 and LC3 in SK-siBmil and SK-siNS cells. The amount of LC3-II correlates with the extent of autophagosome formation. Thus, the conversion of LC3-I to LC3-II serves as a marker for the accumulation of autophagic vesicles and autophagic activity. Following 5-FU treatment, the ratio of LC3-II/LC3-I and the accumulation of LC3-II was increased. The data demonstrates that 5-FU triggers autophagy in SK-HEP-1 cells (Fig. 3). Western blot analysis revealed that knockdown of endogenous Bmi-1 led to an increase in the levels of Beclin-1 and accumulation of LC3-II. The results identified that Bmi-1 depletion enhances 5-FU-induced autophagy in SK-HEP-1 cells.

\section{Discussion}

The overall response rate to systemic chemotherapy for the treatment of HCC is generally less than $10 \%$, owing to drug resistance and advanced stage of disease. 5-FU is one of the most widely used agents in cancer chemotherapy, and enhancing the sensitization of cancer cells to drug-induced apoptosis has become an important strategy for chemotherapy. Overexpressed Bmi-1 was observed in a significant number of HCC cases, which correlated with advanced invasive stage of tumor progression and poor prognosis. The deregulation of Bmi-1 expression has been linked with proliferation and oncogenesis in human cells. Recently, it has been reported that Bmi-1 is also associated with the protection of tumor cells from apoptosis.

This study represents an investigation into the possibility of combining 5-FU treatment and Bmi-1 depletion as a clinical strategy for liver cancer chemotherapy. To examine the role of Bmi-1-mediated chemotherapy-induced apoptosis, the HCC cell lines, SK-HEP-1 and SMMC-7721, in which Bmi-1 is highly expressed, were used in our study. The MTT results demonstrated that depletion of Bmi-1 increased the sensitivity of the cells to 5-FU in SK-HEP-1 cells compared to SMMC-7721, thus, we selected SK-HEP-1 cells for further investigation. FACS revealed that silencing Bmi-1 expression may enhance 5-FU-induced apoptosis in SK-HEP-1 cells.

Constitutive activation of the PI3K/AKT signaling pathway has been firmly established as a major determinant of tumor cell growth and survival in a multitude of solid tumors (12). PI3K subsequently produces the lipid second messenger PIP3b, 
which in turn activates AKT. Activated AKT phosphorylates the Bcl-2-associated death promoter and the inactivation of the Bcl-2-associated death promoter decreases apoptosis and increases cell survival (13). To determine the underlying mechanisms that are not fully understood, we investigated the contribution of Bcl-2 family proteins to 5-FU-induced apoptosis and revealed an increase in the expression of Bax protein and a decrease in the expression of Bcl-2 in SK-HEP-1 cells. Furthermore, we demonstrated that knockdown of Bmi-1 inhibits AKT activation, suggesting an essential role of the PI3K/AKT pathway in the sensitization of Bmi-1 to 5-FU treatment. The knockdown of endogenous Bmi-1 expression contributed to sensitizing HCC cells to the anticancer drug 5-FU by increasing apoptosis.

The connection between autophagy and apoptosis is a developing area of research. For tumors, autophagy is a 'doubled-edged sword'. Its protective function helps tumor cells survive under stress, including certain chemotherapies; however, autophagy may also have a negative impact on cell growth, and autophagy-associated cell death has been demonstrated in response to various anticancer therapies (14). The aim of this study was to investigate whether 5-FU triggers autophagy and whether depletion of Bmi-1 enhances 5-FU-induced autophagy in SK-HEP-1 cells. Our results demonstrate that 5-FU increased the conversion of LC3-I to LC3-II, suggesting that autophagy was induced in 5-FU-treated SK-HEP-1 cells. Beclin-1 is a crucial regulator of autophagy that directly interacts with the anti-apoptotic protein, Bcl-2 (15). Autophagy is induced by the release of Beclin-1 from Bcl-2. We examined whether Beclin-1 signaling is involved in 5-FU-induced autophagy. Western blot analysis demonstrated that treatment of 5-FU led to an increase in the levels of Beclin-1, decrease in the levels of Bcl-2 and accumulation of LC3-II. The results suggested that Bcl-2/Beclin-1 signaling is involved in 5-FU-induced autophagy. We examined the expression of Beclin-1 and LC3 in SK-siBmi1 and SK-siNS cells with or without 5-FU treatment. Western blot analysis revealed that knockdown of endogenous Bmi-1 led to an increase in the levels of Beclin-1 and accumulation of LC3-II. Together, these results indicated that 5-FU triggers autophagy and depletion of Bmi-1 enhanced 5-FU-induced autophagy.

In conclusion, we report for the first time the anticancer potential of a combination of 5-FU treatment and Bmi-1 depletion in HCC cell lines. We identified that the knockdown of Bmi-1 increases the sensitivity of the SK-HEP-1 and SMMC-7721 cells to 5-FU treatment, and that depletion of Bmi-1 enhances 5-FU-induced apoptosis with a concomitantly induced autophagy in SK-HEP-1 cells. The PI3K/AKT and Bcl-2/Beclin-1 signaling pathway was involved in the sensitization of Bmi-1 to 5-FU treatment. Together, the combination of 5-FU and Bmi-1 depletion may be a potential therapeutic strategy in the treatment of human HCC.

\section{Acknowledgements}

This study was supported by the National Natural Science Foundation of China (Nos. 81041083 and 81172778), the Anhui Provincial Natural Science Foundation (No. 1208085QH162) and AUST Grants (Dong $\mathrm{Hu}$ and Jing Wu).

\section{References}

1. Nagano H: Treatment of advanced hepatocellular carcinoma: intraarterial infusion chemotherapy combined with interferon. Oncology 78 (Suppl 1): 142-147, 2010.

2. LIovet JM and Bruix J: Molecular targeted therapies in hepatocellular carcinoma. Hepatology 48: 1312-1327, 2008.

3. Boulin M, Guiu S, Chauffert B, Aho S, Cercueil JP, Ghiringhelli F, Krause D, Fagnoni P, Hillon P, Bedenne L and Guiu B: Screening of anticancer drugs for chemoembolization of hepatocellular carcinoma. Anticancer Drugs 22: 741-748, 2011.

4. Haupt Y, Alexander WS, Barri G, Klinken SP and Adams JM: Novel zinc finger gene implicated as myc collaborator by retrovirally accelerated lymphomagenesis in E mu-myc transgenic mice. Cell 65: 753-763, 1991.

5. van Lohuizen M, Verbeek S, Scheijen B, Wientjens E, van der Gulden $\mathrm{H}$ and Berns A: Identification of cooperating oncogenes in $\mathrm{E}$ mu-myc transgenic mice by provirus tagging. Cell 65: 737-752, 1991.

6. Valk-Lingbeek ME, Bruggeman SW and van Lohuizen M: Stem cells and cancer; the polycomb connection. Cell 118: 409-418, 2004.

7. Gunster MJ, Raaphorst FM, Hamer KM, den Blaauwen JL, Fieret E, Meiier CJ and Otte AP: Differential expression of human polycomb group proteins in various tissues and cell types. J Cell Biochem Suppl 36: 129-143, 2001.

8. Wang H, Pan K, Zhang HK, Weng DS, Zhou J, Li JJ, Huang W, Song HF, Chen MS and Xia JC: Increased polycomb-group oncogene Bmi-1 expression correlates with poor prognosis in hepatocellular carcinoma. J Cancer Res Clin Oncol 134: 535-541, 2008.

9. Liu L, Andrews LG and Tollefsbol TO: Loss of the human polycomb group protein BMI1 promotes cancer-specific cell death. Oncogene 25: 4370-4375, 2006.

10. Wu J, Hu D, Yang G, Zhou J, Yang C, Gao Y and Zhu Z: Down-regulation of Bmi-1 cooperates with artemisinin on growth inhibition of nasopharyngeal carcinoma cells. J Cell Biochem 112: 1938-1948, 2011.

11. Chipuk JE and Green DR: How do Bcl-2 proteins induce mitochondrial outer membrane permeabilization? Trends Cell Biol 18: 157-164, 2008.

12. Whittaker S, Marais $\mathrm{R}$ and Zhu AX: The role of signaling pathways in the development and treatment of hepatocellular carcinoma. Oncogene 29: 4989-5005, 2010.

13. Chen YL, Law PY and Loh HH: Inhibition of PI3K/Akt signaling: an emerging paradigm for targeted cancer therapy. Curr Med Chem Anticancer Agents 5: 575-589, 2005.

14. Gump JM and Thorburn A: Autophagy and apoptosis: what is the connection? Trends Cell Biol 21: 387-392, 2011.

15. Zhou F, Yang Y and Xing D: Bcl-2 and Bcl-xl play important roles in the crosstalk between autophagy and apoptosis. FEBS J 278: 403-413, 2011. 